\title{
Micrographic (MOHS') Surgery in the Management of Periocular Basal Cell Epitheliomas
}

\author{
R. N. DOWNES,${ }^{1}$ N. P. J. WALKER,${ }^{2}$ and J. R. O. COLLIN ${ }^{1}$ \\ London
}

\begin{abstract}
Summary
The use of fresh tissue micrographic surgery offers distinct advantages over other currently available forms of treatment in the management of primary and recurrent periocular basal cell epitheliomas. The technique is described and the results of a prospective study of 22 patients are presented. The advantages in terms of both surety of tumour excision and tissue preservation are discussed. We conclude that micrographic surgery has a definite role to play in the management of certain periocular basal cell epitheliomas.
\end{abstract}

The management of periocular basal cell epitheliomas is aimed at irradication of the disease process coupled with preservation of adjacent healthy tissue. Basal cell carcinomas whilst 'only' locally invasive are potentially lethal tumours. Fifty years ago Birge ${ }^{1}$ reported an $11 \%$ mortality rate; Payne and co-workers ${ }^{2}$ cited a $2 \%$ mortality rate 30 years later. A number of different treatment modalities have been described in the management of basal cell epitheliomas. ${ }^{2-4}$ Curettage and electrodessication, cryosurgery and radiotherapy are reported as achieving five year cure rates in the region of $90 \% .^{2-8}$ The results using conventional surgical excision suggest cure rates in excess of $80 \%{ }^{9,10}$ All of these forms of treatment may therefore be associated with a significant rate of recurrence of the primary tumour. Total tumour irradication is most likely with aggressive initial treatment; successful long-term cure is considerably less after recurrence. Currently the best available treatment options are surgical excision with standard frozen section control and micrographic surgery. Reports suggest five year cure rates using these forms of treatment in excess of $95 \% .4,10-15$

Conventional frozen section control is widely used but in many instances only a small fraction of the periphery of the excised tumour is actually examined microscopically. The fresh tissue technique of micrographic surgery, an adaptation of Mohs' original fixed tissue technique ${ }^{16}$ was developed to overcome just such limitations. Between December 1987 and December 1988, all patients presenting with a periocular tumour to either the Lid Clinic at Moorfields Eye Hospital or the Micrographic Surgery Unit at St John's Hospital have been considered for micrographic surgery. Twenty two cases were felt to be suitable for management using this technique.

\section{Materials and Methods}

Twenty two patients were included in this study; all were managed using micrographic surgery performed jointly by a dermatologist trained in Mohs' surgery (NPJW) and an ophthalmologist (RND) at St John's Hospital for Diseases of the Skin. All patients were evalu-

From: ${ }^{1}$ Moorfields Eye Hospital, London and ${ }^{2}$ St John's Hospital for Diseases of the Skin, London.

Correspondence to: Sqn Ldr R. N. Downes FRCS FCOphth, Consultant Ophthalmic Surgeon, Ophthalmic Department, Princess Mary's Hospital Halton, Royal Air Force Halton, Aylesbury, Bucks HP22 5PG. 
Table I Periocular Location of Basal Cell Epitheliomas

Medial canthal region

Lateral canthal region

Upper eyelid

Lower eyelid

Nasal bridge and medial canthus

Upper eyelid and medial canthus

Lower eyelid and medial canthus

Total

11

2

2

4

1

1

1

22

Table II Pre and Post Micrographic SurgeryTumour and Defect Sizes

a. Initial macroscopic tumour dimensions

Range $8 \mathrm{~mm} \times 6 \mathrm{~mm}-45 \mathrm{~mm} \times 40 \mathrm{~mm}$

Average $17 \mathrm{~mm} \times 12 \mathrm{~mm}$

b. Final defect sizes after micrographic surgery

Range $15 \mathrm{~mm} \times 10 \mathrm{~mm}-55 \mathrm{~mm} \times 50 \mathrm{~mm}$

Average $25 \mathrm{~mm} \times 15 \mathrm{~mm}$

ated pre-operatively by one of us (JROC) who outlined a management plan including likely reconstruction (had micrographic surgery not been available) in each case. The technique of fresh tissue micrographic surgery is well described. ${ }^{11,14}$ All procedures were performed under local anaesthesia. Following initial tumour debulking if appropriate, residual macroscopic tumour and a $1-2 \mathrm{~mm}$ margin of normal tissue were excised as a single layer with notching of the tissue margins to allow for accurate tissue orientation. The tissue layer was divided into pieces of manageable size for histological processing. The cut edges of each piece were stained with permanent coloured dyes to enable precise tissue mapping. Horizontal frozen sections were cut from the under surface of each piece, routinely stained and examined microscopically for evidence of tumour. When residual tumour was present, the exact localisation was known as a result of the tissue mapping; a further tissue layer was excised from any specifically identified areas and the process continued until a tumour free plane was reached.

Reconstruction of the defect was undertaken either at St John's or Moorfields. Immediate repair was performed (RND) in a few cases under local anaesthesia at St John's such that the patient was managed effectively as an outpatient. In the majority of cases reconstruction was performed at Moorfields (JROC or RND) using either local or general anaesthesia within 48 hours of the initial micrographic surgery.

\section{Results}

The results of a prospective study using micrographic surgery in 22 patients with periocular basal cell epitheliomas are presented. Fourteen females and eight males with an age range of 27 to 85 years (average 67 years) were evaluated. Nine patients had recurrent basal cell epitheliomas of which six had previously been managed with radiotherapy, two with surgery and one with combined radiotherapy and surgery. The site of each tumour is shown in Table I. Seven of the nine recurrences involved the medial canthus, one arose in the lower lid and one at the lateral canthus. The number of tissue layers excised micrographically ranged from one to five and number of tissue pieces examined from three to 23 .

The initial tumour sizes and final post micrographic surgery defect sizes are outlined in Table II. Fourteen of the 22 basal cell epitheliomas extended beyond a $4 \mathrm{~mm}$ margin of normal tissue. Preservation of the lacrimal or canalicular apparatus was possible in eight of the 12 cases in whom sacrifice of the lacrimal apparatus had been felt necessary pre-oper-

Table III Surgical Reconstruction

\begin{tabular}{llcc}
\hline & & Anticipated & Actual \\
\hline 1. & Direct closure & 1 & 2 \\
2. & Post-auricular Wolfe graft & 5 & 13 \\
& (PAWG)/simple local flap & & 3 \\
3. & Complicated local flap/PAWG & 9 & 4 \\
& and simple local flap & 5 & - \\
4. & Extensive reconstruction & 2 & \\
5. & Laissez-faire & & \\
\hline
\end{tabular}






Fig. 1. Patient 1-Recurrent basal cell carcinoma of lateral lower lid and cheek.

atively. Similarly significant posterior lamella was preserved in three out of four patients. In comparison with the anticipated reconstruction, nine cases required a simpler repair; one required more extensive reconstruction (Table III). All patients have been followed up for a minimum of six months (range 6-18 months; average 11 months). To date no evidence of tumour recurrence has arisen in any patient.

The following cases are representative.

Case 1. This patient had a recurrent basal cell carcinoma, treated in the past with both surgery and radiotherapy (Fig. 1). Micrographic surgery was performed leaving a final defect as shown in Figure 2 but preserving the posterior lamella of the lower lid in toto. Figures 3 and 4 demonstrate the reconstruction using a rotation flap as shown. Extensive reconstruction of the posterior lamella was not required thus simplifying the surgical procedure whilst improving the final reconstructional result in terms of both cosmesis and function.

Case 2. This patient had a medial canthal basal cell epithelioma, (Fig. 5). Preservation of the lacrimal apparatus was possible using micrographic surgery, (Fig. 6). Figures 7 and 8 demonstrate the postoperative appearances.

\section{Complications}

In two patients a single focus of tumour apparently remained after micrographic surgery, which was abandoned prematurely in each case. One patient experienced severe discomfort after excision of a single layer and further surgery was felt inappropriate. The procedure was terminated in the second patient for logistics reasons. The remaining focus of tumour in each case overlay the lacrimal sac. Both patients underwent lacrimal sac excision under general anaesthesia prior to reconstruction. Formal histology failed to identify significant residual tumour in either case.

\section{Discussion}

Periocular basal cell epitheliomas are the most common malignant tumours encountered by the ophthalmologist. The potentially lethal nature of these lesions should not be forgotten when considering the best form of management. A recurrence rate in the region of $10 \%$ as reported using certain modes of treatment $^{2-8}$ is unacceptable for primary eye- 


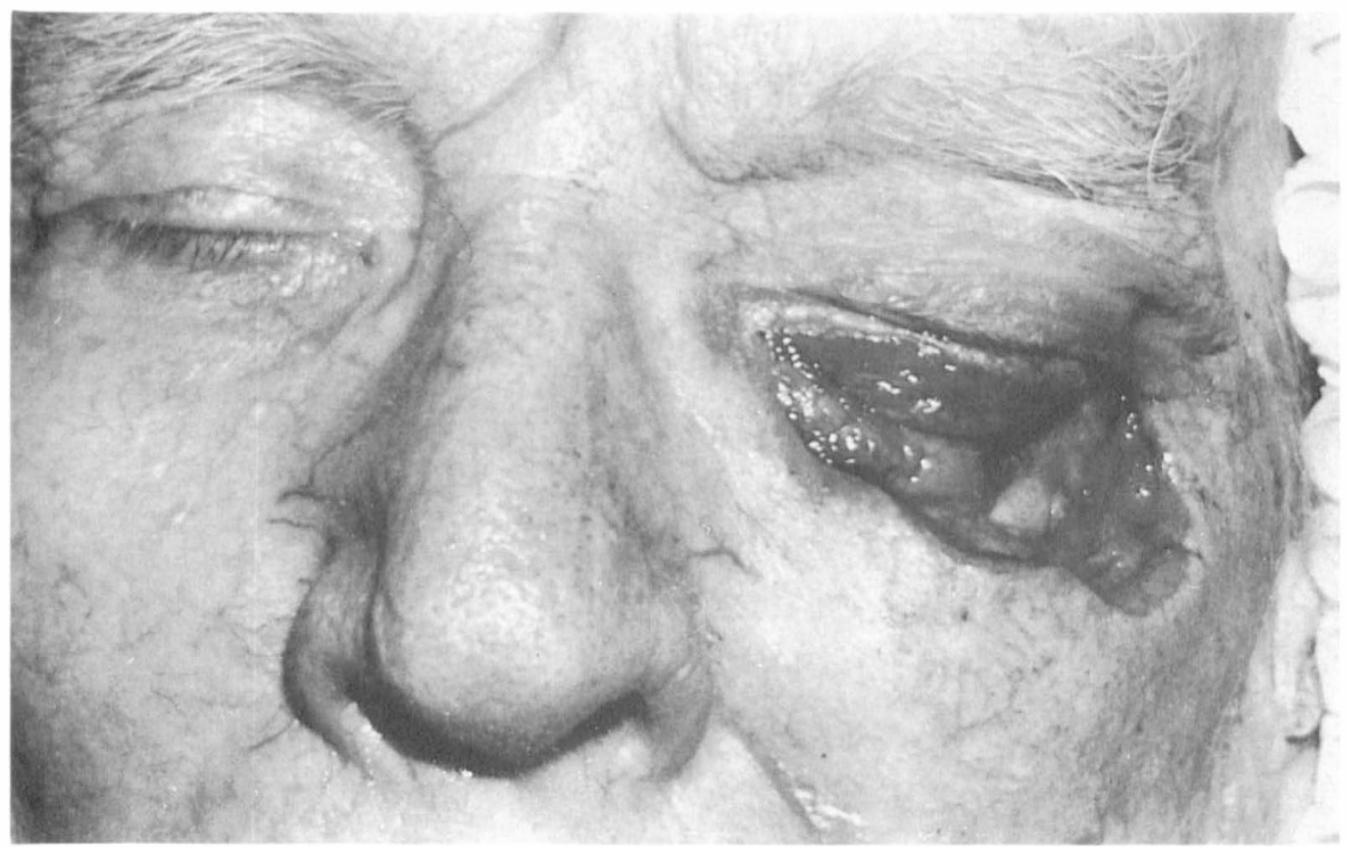

Fig. 2. Patient 1-Appearances following micrographic (Mohs') surgery with preservation of posterior lamella of lower lid.

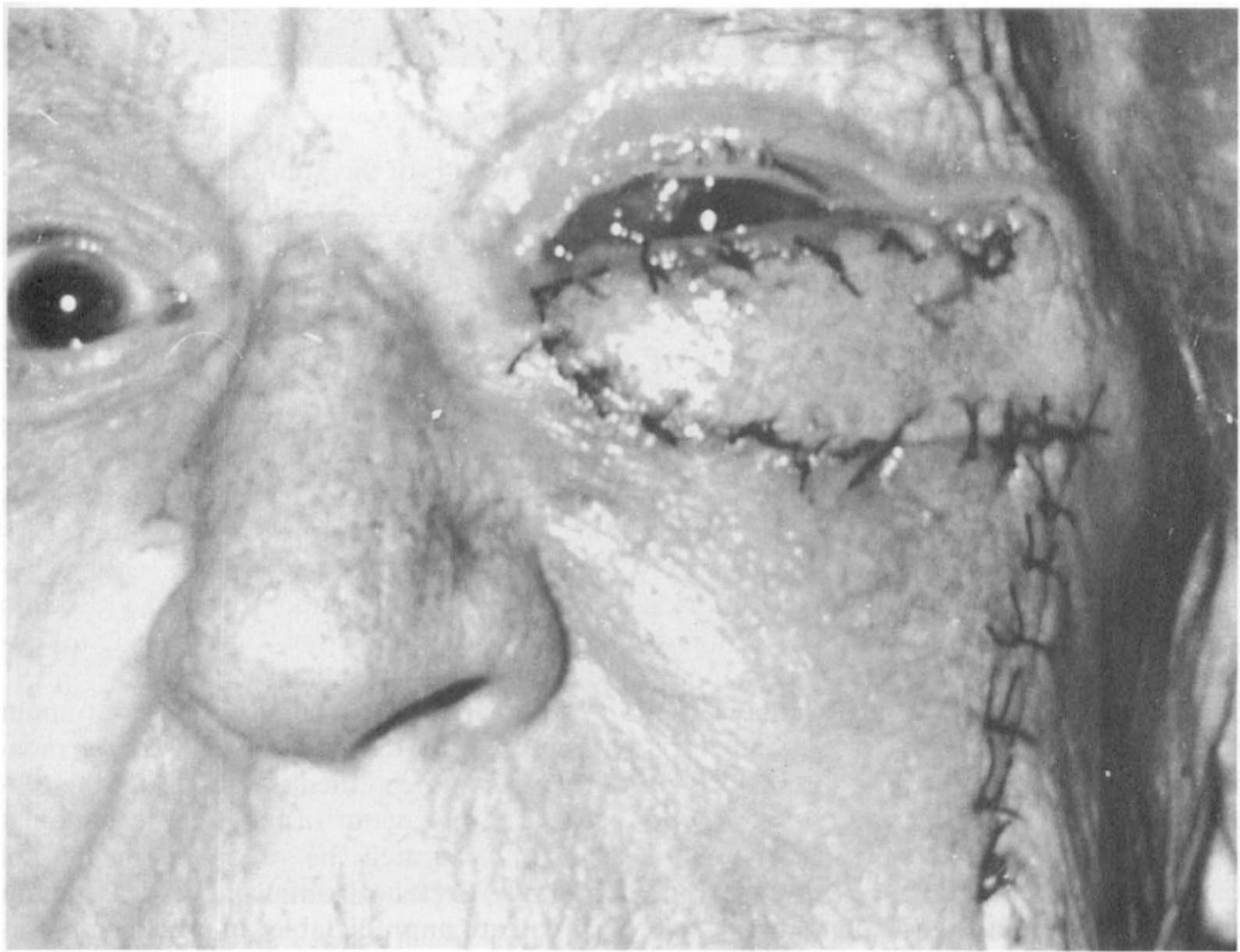

Fig. 3. Patient 1-Reconstruction using cheek rotation flap. 


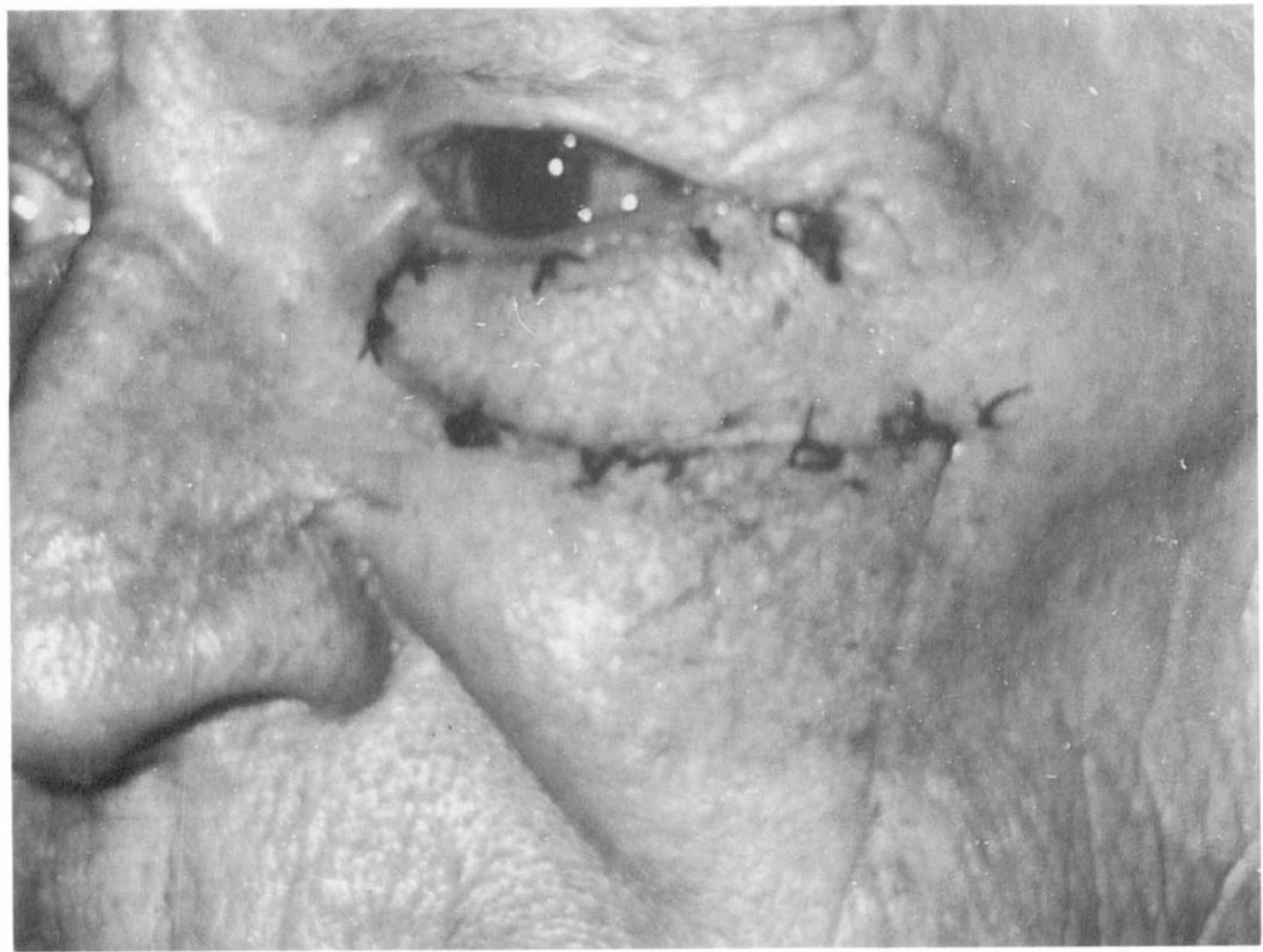

Fig. 4. Patient 1-Appearances one week post operatively.

lid tumours. Nowhere in the body are basal cell epitheliomas more dangerous or are the functional and cosmetic sequelae of excess tissue removal more damaging, than in the periocular tissues. ${ }^{13}$

Tumour excision with either conventional frozen section control or micrographic surgery produces higher cure rates than all other forms of treatment currently available., ,9-13 $^{-1}$ Although the former is widely used in this country, the use of micrographic surgery in the management of periocular basal cell epitheliomas has not previously been reported here. The micrographic (Mohs') technique offers considerable advantages over standard frozen section monitoring. The available literature suggests a higher cure rate and the technique is more conservative in terms of tissue sparing. A number of American authors ${ }^{9,11,13,17}$ now recommend fresh tissue micrographic surgery as the definitive treatment for certain periocular basal cell epitheliomas.
The results of this study confirm the advantages of micrographic surgery. Had conventional surgery with a $4 \mathrm{~mm}$ 'safe' margin been used then $64 \%$ of the tumours would have been incompletely excised. Of these tumours ten were primary lesions, four of which were comparatively small (less than $10 \mathrm{~mm}^{2}$ ). Whilst the majority of these tumours were clinically and/or histologically morphoeic some of the smaller lesions were clinically localised and histologically solid or cystic variants. Thus conventional surgery without histological control cannot be relied upon to effect a cure even in small apparently well circumscribed basal cell epitheliomas.

Comparison regarding certainty of tumour excision with standard frozen section monitoring is more difficult and largely theoretical. The behaviour of basal cell carcinomas cannot accurately be predicted on clinical grounds. Both horizontal and vertical tumour spread are unpredictable. In practice the surgeon 'guides' the histopathologist in conven- 




Fig. 5. Patient 2-Basal cell epithelioma of medial canthus.

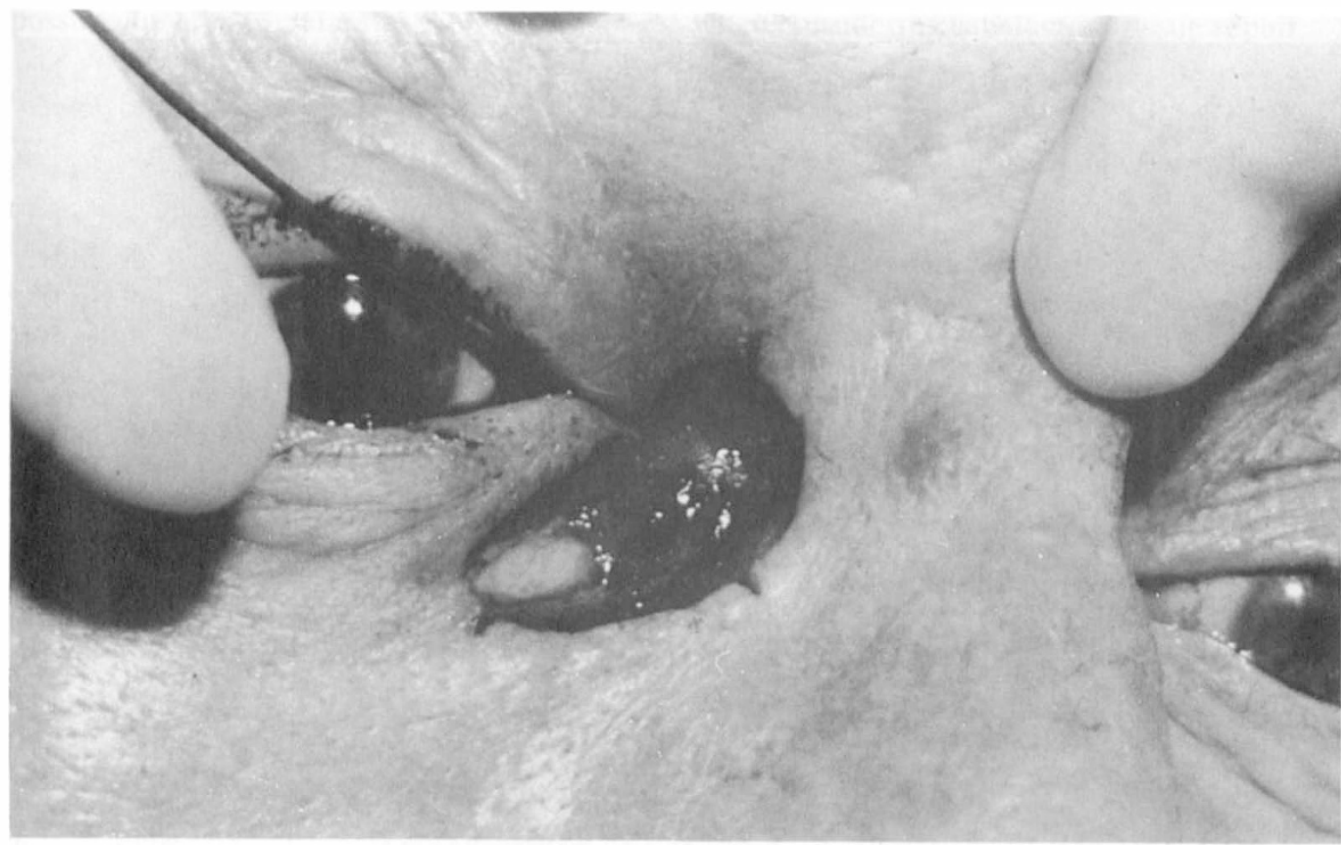

Fig. 6. Patient 2-Appearances following micrographic (Mohs') surgery-lacrimal probe in inferior canaliculus demonstrating canalicular preservation. 


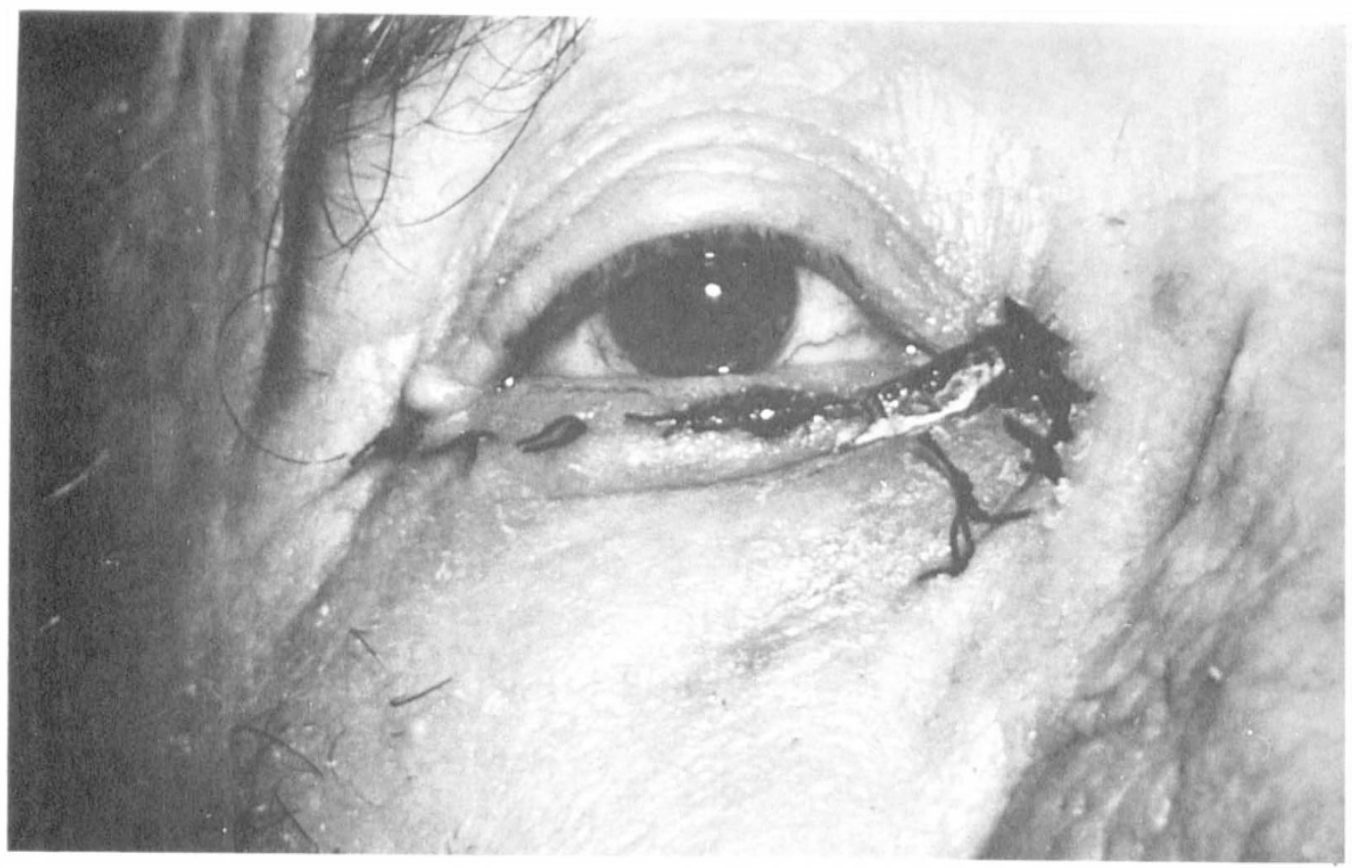

Fig. 7. Patient 2-Post reconstruction using advancement flap.

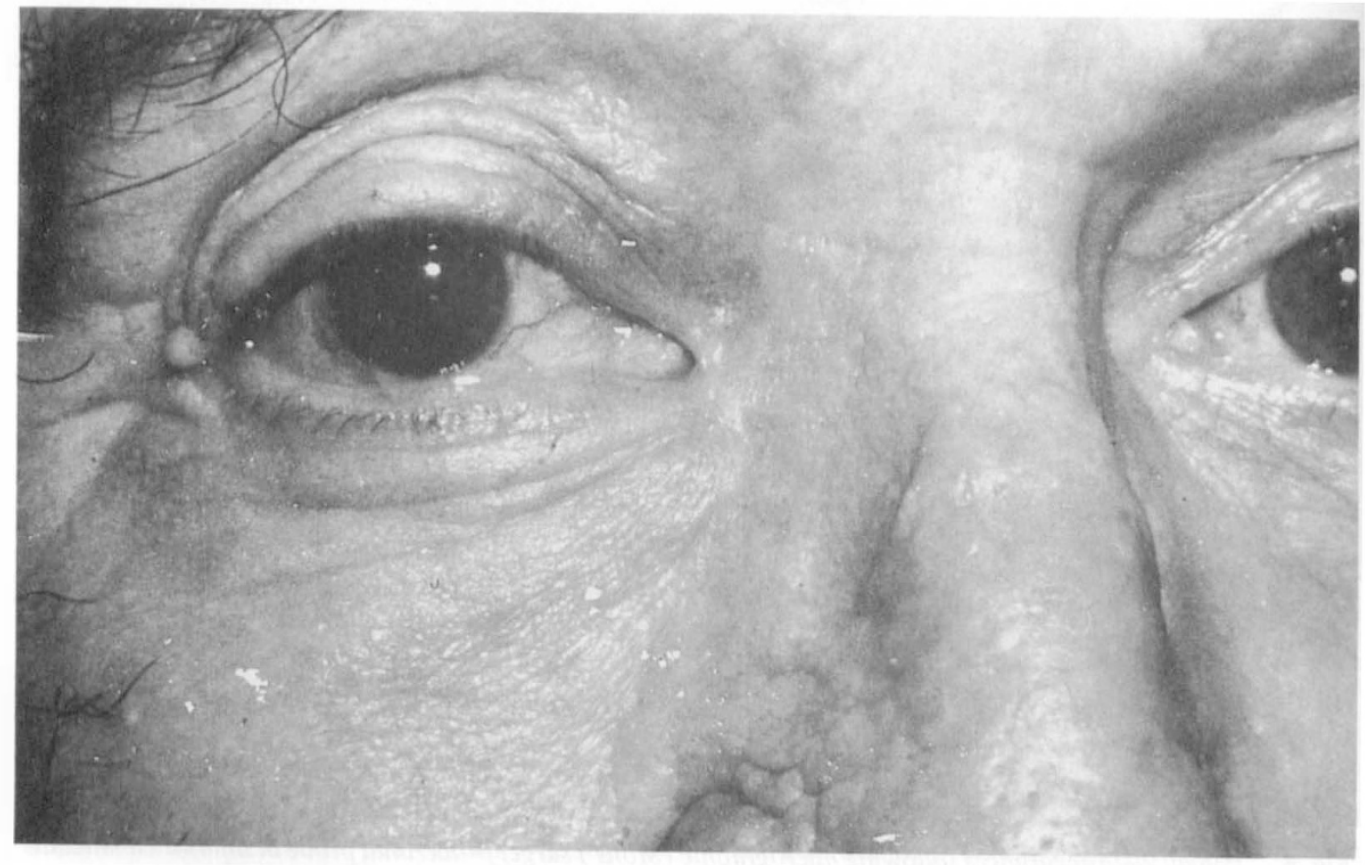

Fig. 8. Patient 2-Post operative appearances three months after surgery. 
tional frozen section monitoring such that only certain margins or surfaces of excised tissue are examined for tumour. Had this policy, as opposed to complete sectioning and microscopic examination of all excised tissue, been adopted then certainly as a result of erratic tumour behaviour excision would have been incomplete in some cases.

The micrographic technique converts an essentially two -dimensional view (as seen with the standard frozen section) into a threedimensional picture by the use of accurate segmental tissue mapping. Only those areas showing residual tumour rather than entire margins or surfaces are sequentially excised, allowing maximal preservation of healthy tissue. This advantage is illustrated in some of our patients. Preservation of part or all of the lacrimal apparatus, which would otherwise have been sacrificed, was possible in $67 \%$ of patients. Preservation of significant posterior lamella in three patients (two upper eyelids and one lower eyelid) made subsequent reconstruction of a relatively normal eyelid possible without resort to free grafts. A simpler reconstruction in terms of either the surgical procedure or the duration of surgery coupled with local or general anaesthesia was possible in $41 \%$ of patients.

Micrographic surgery is not however without disadvantages. The patient may not tolerate the procedure under local anaesthesia for a variety of reasons. Adequate anaesthesia is often difficult to achieve in scarred tissue because of previous radiation therapy or surgery. In spite of seemingly adequate local anaesthesia, one patient in our series found the procedure most uncomfortable. The tumour was a large recurrent lesion at the medial canthus which previously had been treated with combined radiotherapy and curettage. Underlying fibrosis and scarring were widespread. It was felt that as a result of this tissue distortion coupled with poor anaesthesia of the periosteum, further surgery should be performed under general anaesthesia. The majority of patients are elderly; some are frail. Repeated tissue excisions over the course of several hours may be particularly arduous for the patient and surgeon alike! The process of micrographic surgery can be quite time consuming and the facility is not generally available in this country. We were forced to abandon micrographic surgery in a second patient as a result of lack of theatre time coupled with support staff shortages. Since a single focus only of possible tumour remained the decision was taken to complete the surgical excision as a planned rather than reschedule for both further micrographic surgery and subsequent reconstruction.

Both patients in whom the procedure was abandoned prematurely had excision of the lacrimal sac in view of the probable residual tumour. Formal histology in both cases failed to demonstrate histological evidence of significant tumour.

The initial results obtained in this study have been most encouraging, although the duration of patient follow-up to date is relatively short. It would appear that micrographic surgery has a definite role to play in the management of certain periocular tumours. We recommend that fresh tissue micrographic surgery is the procedure of choice in the management of tumours involving the medial canthal region, tumour recurrences and tumours involving periorbital regions in which tissue preservation eg posterior lamella of the upper eyelid is of particular importance when considering satisfactory tissue repair.

We are particularly grateful to $\mathrm{Mr} \mathrm{D}$. Ramnarain, whose expert technical assistance was invaluable to this study, and Miss G. Mason for her secretarial support.

\section{References}

${ }^{1}$ Birge HL: Cancer of the eyelids: I. Basal cell and mixed basal cell and squamous cell epitheliomas. Arch Ophthalmol 1938, 19: 700-8.

${ }^{2}$ Payne JW, Duke JR, Butner R, Eifzig DE: Basal cell carcinoma of the eyelids: A long term follow up study. Arch Ophthalmol 1969, 81, 553-8.

${ }^{3}$ Wiggs EO: Incompletely excised basal cell carcinoma of the ocular adnexae. Ophthalmic Surg 1981, 12: 891-6.

${ }^{4}$ Rodriguez-Sains RS and Jakobiec FA: Eyelid and conjunctival neoplasms. In Smith BC, Della Rocca RC, Nesi FA and Lisman RD eds. Ophthalmic Plastic and Reconstructive Surgery. St Louis: CV Mosby 1987, 760-2.

${ }^{5}$ Gladstein AH: Efficacy, simplicity and safety of x-ray therapy of basal cell carcinomas on periocular skin. J Dermatol Surg Oncol 1978, 4: 586-93.

${ }^{6}$ Kopf AW, Bart RS, Schraeger D, Lazar M, Popkin GL: Curettage and electrodessication treatment 
of basal cell carcinoma. Arch Dermatol 1977, 113: 439-43.

${ }^{7}$ Gladstein AH: Radiotherapy of eyelid tumours. In Jakobiec FA ed. Ocular and Adnexal Tumours. Birmingham: Aesculapius, 1978, 508-16.

${ }^{8}$ Fraunfelder FT, Zacarian SA, Limber BL, Winefeld D: Cryosurgery for malignancies of the eyelid. Ophthalmology 1980, 87: 461-5.

${ }^{9}$ Aurora AL and Blodi FC: Reappraisal of basal cell carcinoma of the eyelids. Am J Ophthalmol 1970, 70: 329-36.

${ }^{10}$ Chalfin J and Putterman AM: Frozen section control in the surgery of basal cell carcinoma of the eyelid. Am J Ophthalmol 1979, 87: 802-9.

${ }^{11}$ Anderson RL and Ceilley RI: A multispeciality approach to the excision and reconstruction of eyelid tumours. Ophthalmology 1978, 85: 1150-63.
${ }^{12}$ Doxanas MT, Green WR, Iliff CE: Factors in the successful management of basal cell carcinomas of the eyelids. Am J Ophthalmol 1981, 91: 726-36.

${ }^{13}$ Anderson RL: Mohs' micrographic technique. Arch Ophthalmol 1986, 104: 818-9.

${ }^{14}$ Mohs FE: Micrographic surgery for the microscopically controlled excision of eyelid cancers. Arch Ophthalmol 1986, 104: 901-9.

${ }^{15}$ Rodriguez-Sains RS, Robins P, Smith B, Bosniak SL: Radiotherapy of periocular basal cell carcinomas: recurrence rates and treatment with special attention to the medial canthus. Br JOpthalmol 1988, 72: 134-8.

${ }^{16}$ Mohs FE: Chemosurgery: a microscopically controlled method of cancer excision. Arch Surg 1941, 42: 279-95.

${ }^{17}$ Robins P: Chemosurgery, my 15 years experience. J Dermatol Surg Oncol 1981, 7: 779-89. 